\title{
Knowledge of Objective Modality
}

\author{
Margot Strohminger and Juhani Yli-Vakkuri
}

\begin{abstract}
The epistemology of modality has focused on metaphysical modality and, more recently, counterfactual conditionals. Knowledge of kinds of modality that are not metaphysical has so far gone largely unexplored. Yet other theoretically interesting kinds of modality, such as nomic, practical, and 'easy' possibility, are no less puzzling epistemologically. Could Clinton easily have won the 2016 presidential election - was it an easy possibility? Given that she didn't in fact win the election, how, if at all, can we know whether she easily could have? This paper investigates the epistemology of the broad category of 'objective' modality, of which metaphysical modality is a special, limiting case. It argues that the same cognitive mechanisms that are capable of producing knowledge of metaphysical modality are also capable of producing knowledge of all other objective modalities. This conclusion can be used to explain the roles of counterfactual reasoning and the imagination in the epistemology of objective modality.
\end{abstract}

The epistemology of modality has focused almost exclusively on knowledge of metaphysical modality. ${ }^{1}$ However, other kinds of so-called objective modality (in the sense of Williamson $2016 b$ ) such as nomic, practical, and 'easy' modality can also appear epistemologically puzzling, and they are important topics in their own right. Thus the neglect of the epistemology of other objective modalities may look unmotivated or parochial. At worst, it may look similar to an approach to the epistemology of mathematics that only deals with knowledge of some very weak mathematical theory, such as Robinson arithmetic.

This paper makes a start on a more comprehensive approach to the epistemology of modality, of which metaphysical modality is a special, limiting case. Knowledge of other objective modalities and knowledge of metaphysical modality are puzzling in many of the same ways. It will be argued that by and large the same cognitive mechanisms that are capable of producing knowledge of metaphysical modality - in particular, those we use for acquiring knowledge of counterfactuals - are also capable of producing knowledge of all other objective modalities. This idea is anticipated by Williamson in his classic discussion of the central role of counterfactuals in the epistemology of metaphysical modality, where he says, in passing, that

the connections [of metaphysical possibility] with restricted [objective] possibility and with counterfactual conditionals are not mutually exclusive, for they are not being interpreted as rival semantic analyses, but rather as different cases in which the cognitive mechanisms needed for one already provide for the other (2007: 178).

Given that all objective modalities are restrictions of metaphysical modality, it should not be surprising that the relationship between the epistemologies of objective modality and metaphysical modality turns out to be more like that between the epistemologies of restricted

\footnotetext{
${ }^{1}$ See Strohminger and Yli-Vakkuri (2017) for review.
} 
quantification and unrestricted quantification than that between the epistemologies of Robinson arithmetic and all arithmetic.

\section{The epistemology of objective modality}

Some modalities are restrictions of metaphysical modality-following Williamson, we will call these the objective modalities. ${ }^{2}$ Metaphysical modality can be defined (following Williamson) in terms of restricted objective modality: a proposition is metaphysically necessary iff it is necessary in every objective sense (Williamson 2016b: 455). (Objective modalities can also be defined in terms of metaphysical modality, as we will see in $\$ 2$.) Thus whatever is metaphysically necessary is also necessary in any objective sense, and whatever is possible in some objective sense is also metaphysically possible. An objective modality that is restricted by a trivial condition, such as a truth-functional tautology, is still an objective modality. Metaphysical modality thus counts as a trivial restriction of itself, and so counts as an objective modality.

Nomic (or nomological) modality is a paradigm case of a non-trivially restricted objective modality. Many of the objective modalities we express using the modal words ('possibly', 'necessarily', 'actually', 'could', 'would', etc.) outside of theoretical contexts are far more restricted than that paradigm. They include, inter alia, 'practical' and 'easy" ${ }^{3}$ varieties of modality. When we ask whether it is practically possible that the Democrats won the 2016 U.S. presidential election, we are asking a question equivalent to this: is it metaphysically possible given the practical constraints that the Democrats won the 2016 U.S. presidential election? What counts as a 'practical constraint' depends on the context. In this case they may include, for example, the fact that Trump was the Republican nominee, and in every case they will include the (actual) laws of nature. And to ask whether something is easily possible — or, to use a more colloquial idiom, whether something could easily have happened - is roughly equivalent to asking whether it is metaphysically possible given that things are similar or close to how they actually are. What counts as 'similar' or 'close' also depends on context, but in any actual context 'similarity' to actuality requires sameness with respect to the laws of nature.

Not everything we express using the modal words is a restriction of metaphysical modality. So-called epistemic modalities are a paradigm example. Both the Generalized Continuum Hypothesis and its negation are epistemically possible, since neither is known, but one of them is metaphysically impossible. Epistemic 'modalities' do not even seem to be modalities in that they are not properties of propositions: ${ }^{4}$ it seems that one and the same proposition can be both epistemically possible when expressed by one sentence and epistemically impossible when expressed by another. For example, it is highly plausible that

\footnotetext{
${ }^{2}$ Williamson is, of course, far from being the only philosopher to recognize a broad category of non-epistemic modalities that includes metaphysical modality: see, for example, Lange (2009), Hale (2013), Kment (2014) and Vetter (2015). Linguists have recognized a similar-perhaps the same - category of 'root', 'circumstantial', or 'dynamic' modality since the 1970s (Kratzer 1981, 2012, Portner 2009).

${ }^{3}$ See Sainsbury (1997), Peacocke (1999: 310-328), and Williamson (2000: 123-130).

${ }^{4}$ Are all and only objective modalities properties of propositions? This seems to us more a matter to be decided than discovered. We think of the objective modalities as all and only those that can be characterized by a restricting condition in the sense of $\S 2$. This does not include all properties of propositions, since there are at least as many properties of propositions as there are functions from metaphysically possible words to propositions: such a function $\sigma$ characterizes the (or a) property $P$ such that a proposition $p$ has $P$ at $w$ iff $p \in \sigma(w)$. The alternative notion of an objective modality as a property of propositions is adequately captured by Scott-Montague 'neighborhood semantics', in which a modal operator is interpreted by an assignment of a set of sets of worlds, thought of as the set of relevantly necessary propositions, to each world. As we point out in $\S 2$, our own approach is equivalent to a relational (or 'Kripke') approach to the semantics of modal logic. Unlike neighborhood semantics, relational semantics cannot interpret a modal operator by an arbitrary property of propositions (see Bull and Segerberg 1984: §21).
} 
the sentence 'It might be that something is Greek and not Hellenic, but it might not be that something is Greek and not Greek', where the 'might' is epistemic, is true in some contexts, even though the proposition that something is Greek and not Hellenic is none other than the proposition that something is Greek and not Greek. Logical 'modality' is even more clearly not a modality (and so a fortiori not an objective modality): it is logically necessary that $p$ just in case the sentence ' $p$ ' is true under all interpretations of its non-logical constants. Thus, for example, it is logically possible that something is Greek and not Hellenic but not logically possible that something is Greek and not Greek, because 'Greek' and 'Hellenic' are non-logical constants. Deontic modality is an unclear case. Since deontic 'must' and 'may' statements don't display any of the hallmarks of non-objectivity, it is tempting to classify them as expressing restrictions of metaphysical modality, but there are also reasons not to rush to judgment here: primarily, evidence suggestive of their non-normality (in the logical sense) and hyperintensionality. ${ }^{5}$ (However that may be, it will turn out, on our analysis, that there are objective modal operators that are deontic in the sense of being restricted by the fulfilment of obligations.)

The epistemology of modality should study all knowledge of objective modality and not only the limiting case of knowledge of metaphysical modality. The central questions in the epistemology of metaphysical modality tend to generalize to all non-trivial objective modalities. For example, the familiar question, 'How, if at all, can we know whether it is metaphysically possible that $p$ when it is not true that $p$ ?' remains puzzling when we replace 'metaphysically' with 'nomically', 'practically', 'easily', 'technologically', etc. (cf. Williamson 2016b: 462). After all, knowledge of such facts is just one valid inferential step away from knowledge of metaphysical possibility: if one knows that it is in some objective sense possible that $p$, one can come to know by deduction that it is metaphysically possible that $p$.

The importance of various restricted objective modalities to quotidian concerns, engineering, policy planning, and planning and decision making in general contributes to an interest in their epistemology. In these contexts, we often use modal words to express restricted objective modalities. In various theoretical contexts, too, we are often interested in whether something is objectively possible in a restricted sense. Epistemology itself is a salient example: in that field the expressions 'reliable', 'knowable', 'risk', 'in a position to know', 'safe', and 'sensitive' are regularly used to express some kind of restricted objective modal notion. For example, it is initially plausible that one is in a position to know only if (and perhaps also if) one can know, in some objective sense of 'can'. ${ }^{6}$ Natural science is concerned with nomic possibility, as well as with other objective modalities whose importance to natural scientific inquiry has gone largely unnoticed in the epistemology of modality until recently (Williamson $2016 b, 2016 c$ ). The 'can' or 'cannot' in standard formulations of Heisenberg's uncertainty principle expresses some kind of objective modality. Even pure mathematics is rife with conjectures, axioms, proofs, and theorems that manifest commitments to various objective modal claims. Typically these commitments are implicit (Yli-Vakkuri and Hawthorne MS $b$ ), but there are also some plausible examples of explicit objective modal commitments in pure mathematics: for example, it seems plausible that Church's thesis concerns computability, or what can be computed, in some objective sense of 'can'.

\section{Objective modalities as restrictions of metaphysical modality}

\footnotetext{
${ }^{5}$ See Fine (2014) for discussion.

${ }^{6}$ See Yli-Vakkuri and Hawthorne (MSa) for discussion.

${ }^{7}$ Thanks to Timothy Williamson for this example. See Yli-Vakkuri and Hawthorne (MS $b$ : note 5) for discussion.
} 
We have already seen that metaphysical modality can be defined in terms of objective modality. This section asks about the other direction: can objective modality be defined in terms of metaphysical modality? (The answer is 'Yes.') Our motivation here is epistemological. After offering a definition, we will discuss how it enables us to extend certain familiar observations in the epistemology of metaphysical modality to the less explored territory of the epistemology of objective modality. ${ }^{8}$

Statements about what is possible or necessary in some (objective ${ }^{9}$ ) sense are closely related to certain statements about what is metaphysically possible or necessary. In particular, when evaluated in the same context, any statement of the form (1) is necessarily equivalent to the corresponding statement of the form $\left(1^{\prime}\right)$, and any statement of the form (2) is necessarily equivalent to the corresponding statement of the form $(2$ '), where ' $R$ ' expresses the property of being the conjunction of all of the conditions that restrict 'possible' and 'necessary' in the context.

It is possible that $p$.

It is metaphysically compossible with the $R$-condition that $p$.

It is necessary that $p$.

It is a metaphysically necessary consequence of the $R$-condition that $p$.

For example, if (1) and (2) express nomic modality, ' $R$ ' in $\left(1^{\prime}\right)$ and $\left(2^{\prime}\right)$ will express the property of being the conjunction of all laws of nature. The restricting conditions corresponding to practical and easy varieties of modality are conjunctions of some highly local conditions, and what ' $R$ ' expresses in these cases is highly sensitive to the context of speech. In the limiting case, where (1) and (2) express metaphysical modality, ' $R$ ' will express the property of being the conjunction of some necessary truths (or of no conditions at all; see below).

It bears emphasis that our assumption about the necessary equivalence of (1) and (1') and of (2) and (2') in any context does not commit us to the view that the condition that restricts a restricted modal operator is something competent users of the operator are able to articulate or express in any way other than by using that very operator. (In this respect restricted modal operators resemble implicitly restricted quantifiers.) In some cases it is fairly easy to specify the restricting condition very precisely using other words: the phrase 'the conjunction of all laws of nature' does the job in the case of nomic modality. But in other cases it is not easy: 'the conjunction of the practical conditions' is extremely vague and uninformative - it is at best a stand-in for a fuller specification of the restricting condition, which we are rarely able to supply. And in some cases it is difficult to come up with any words that even gesture in the right direction (what condition restricts an 'easy' possibility operator?). In cases of the latter kind, it is more natural to think of the restriction associated with the operator as an accessibility relation: a binary relation $\mathbf{R}$ on worlds, such that 'It is necessary that $p$ ' is true at a world $w$ just in case ' $p$ ' is true at every world $v$ such that $w \mathbf{R} v$. In the case of easy possibility, the accessibility relation is some variety of closeness or similarity. But there is no need to have both restricting conditions and accessibility relations, as long as we are working with standard possible-worlds

\footnotetext{
${ }^{8}$ In doing so, we retread some ground covered by van Fraassen (1977), Humberstone (1981), and Hale and Leech (2017). None of these authors propose the analysis we give. Although there is a certain superficial similarity between our analysis and Hale and Leech's, there is also an important difference: see note 11.

${ }^{9}$ Since we are only concerned with objective modalities in this paper, we will henceforth leave the 'objective' implicit.
} 
semantics. Conditions can be represented by accessibility relations, and conversely: given an accessibility relation $\mathbf{R}$, we can define the condition that restricts the modal operators at a world $w$ as $\{v \mid w \mathbf{R} v\}$, and given an assignment of restricting conditions to worlds, we can define the restricting accessibility relation as the relation $\mathbf{R}$ such that $w \mathbf{R} v$ iff $v \in r$, where $r$ is the condition that restricts the modal operators at $w$, i.e., the proposition that is $R$ in $w .^{10}$

The necessary equivalence of statements of forms (1) and (2) in all contexts suggests the following analysis of restricted necessity in terms of metaphysical necessity, which we will assume in what follows. ${ }^{11}$

('It is $R$-necessary that $p$ just in case there is a proposition that is $R$ and $p$ is a metaphysically necessary consequence of it.')

While English does not have special modal words that express metaphysical modality in every context, it is convenient to have ones that do, and for that reason we will use $\square$ and $\diamond$ as context-insensitive operators that express, respectively, metaphysical necessity and possibility, and $\square_{R}$ and $\diamond_{R}$ as schematic necessity and possibility operators restricted by the $R$-condition. So interpreted, it is plausible that, and we will assume that, $\left(\square_{R}\right)$ is logically valid.

In $\left(\square_{R}\right), \square_{R}$ may be interpreted as expressing any restricted necessity. $R$ expresses a property of propositions: the property of being the condition that restricts the necessity operator. The reader should think of $R(q)$ as having the form ' $q$ is the conjunction of propositions $p$ such that ...'. For example, if $\square_{R}$ expresses nomic necessity, then $R$ expresses the property of being the conjunction of the laws of nature, and if $\square_{R}$ expresses metaphysical modality, then $R$ expresses some trivial restriction, such as the property of being the conjunction of the empty set. (We assume that every set of propositions has a conjunction. Consequently, the conjunction of the empty set is a necessary truth: necessarily, all of its conjuncts are true.) We also assume that, whatever property of propositions $R$ may express, it

\footnotetext{
${ }^{10}$ Here we are assuming a coarse-grained conception of propositions (conditions) as sets of worlds, but the intertranslatability of accessibility relation talk with restricting condition talk does not require that assumption. As long as, for each set of worlds $W$, there is a proposition $f(W)$ that is true at exactly the worlds in $W, f(W)$ can play the role of the restricting condition that holds at exactly the worlds in $W$. And it does seem plausible, even given a view on which propositions have arbitrarily fine-grained structure, that there is a function $f$ that fits this description: $f(W)$ might be, for example, the proposition that at least one of the worlds in $W$ is actualized, where 'actualized' is understood in a non-rigid way, so that it is contingent which world is actualized. (Note that we are not assuming that, for each set of worlds $W$, there is a unique proposition that is true at exactly the worlds in $W$. That would be implausible on a structured-propositions view. The axiom of choice guarantees the existence of a suitable function $f$ even if there are sets of worlds that exactly verify more than one proposition. Nor are we assuming the consistency of views on which propositions are arbitrarily fine-grained-theories that posit extremely fine-grained propositional structure are inconsistent: see Dorr 2016a and Goodman 2017. Given an inconsistent view, anything whatsoever is the case.)

${ }^{11}$ Hale and Leech (2017: §6.3) propose an analysis of what they call 'alethic' modality that is superficially similar to but importantly different from $\left(\square_{R}\right)$, which they illustrate with the case of nomic or (as they call it) 'physical' modality, as follows.
}

H\&L: $\quad$ It is physically necessary that $p={ }_{\text {def }} \exists q(\pi(q) \wedge \square(q \rightarrow p))$.

Here ' $\pi$ ' is to be read as 'it is a law of physics that...' (Hale and Leech 2017: 13). This, however, is not an adequate analysis because it does not give the restricted modality being analyzed a normal modal logic: it validates neither necessitation ( $p \rightarrow \square_{R} p$, where $p$ is valid) nor the $\mathbf{K}$ axiom $\left(\square_{R}(p \rightarrow q) \rightarrow\left(\square_{R} p \rightarrow \square_{R} q\right)\right.$ ). Hale and Leech try to solve this problem in a footnote (note 23). We don't think their solution works, but we'll save our criticisms of it for another occasion (Yli-Vakkuri plans to defend his own higher-order analysis elsewhere). 
is necessary that, and it is a logical truth that it is necessary that, there is a unique proposition that has it. That is, we assume that

\section{$\square \exists ! q R(q)$}

and therefore $\square \exists q R(q)$, is valid. (Think of $R(q)$ as having the standard form $q=\wedge\{p \mid \varphi(p)\}$, where $\wedge$ is an infinitary conjunction operator. ${ }^{12}$ )

What about the restricted possibility operator $\diamond_{R}$ ? We will define it as the dual of $\square_{R}$. Because $\square$ and $\diamond$ are duals, validity of

$\left(\diamond_{R \text {-dual }}\right) \quad \diamond_{R} p \leftrightarrow \neg \exists q(R(q) \wedge \square(q \rightarrow \neg p))$

follows immediately. Because $\left(\diamond_{R \text {-dual }}\right)$ and $\square \exists ! q R(q)$ are both valid, so is

$$
\diamond_{R} p \leftrightarrow \exists q(R(q) \wedge \diamond(q \wedge p))
$$

We will treat $\left(\diamond_{R}\right)$ rather than $\left(\diamond_{R \text {-dual }}\right)$ as the canonical equivalence relevant to $\diamond_{R}$ : it captures the intuitive idea that to be restrictedly possible is to be compossible with the restriction.

It is important to read, as we have done, 'the restriction' as having wide scope when it occurs in our informal glosses of restricted necessity and possibility: 'to be restrictedly necessary is to be a necessary consequence of the restriction' and 'to be restrictedly possible is to be compossible with the restriction'. Consider the narrow-scope alternative to $\left(\square_{R}\right)$ :

$\left(\square_{R \text {-nar }}\right) \quad \square_{R} p \leftrightarrow \square \exists q(R(q) \wedge(q \rightarrow p))$.

( $\square_{R \text {-nar }}$ ) is incorrect, as can be seen, for example, by interpreting $\square_{R}$ as expressing practical necessity. Necessarily, the conjunction of the practical conditions is true $(\square \exists q(R(q) \wedge q))$, so by ( $\left.\square_{R \text {-nar }}\right)$, something is practically necessary if and only if it is metaphysically necessary $\left(\diamond_{R}\right.$ $p \leftrightarrow \diamond p)$. ( $\left.\diamond_{R \text {-nar }}\right)$ collapses all modalities for which $\square \exists q(R(q) \wedge q)$ holds-which is to say, all of the factive or T-modalities - into metaphysical modality.

Nor would it do to simulate wide scope for 'the restriction' by restricting metaphysical modality by a sentence that expresses the same restricting condition relative to every metaphysically possible world. To do this, we would have to say that being restrictedly necessary is simply being a necessary consequence of $r$, where $r$ is the restricting condition, i.e.,

$\left(\square_{R \text {-rig }}\right) \quad \square_{R} p \leftrightarrow \square(r \rightarrow p)$,

where $r$ expresses the unique condition that satisfies $R$.

( $\square_{R \text {-rig }}$ ) inappropriately rigidifies restricted modality. Given that the logic of metaphysical modality is $\mathbf{S 5}$ (in which all iterated modalities collapse) the validity of ( $\square_{R \text {-rig }}$ ) entails the validity of both the $4\left(\square_{R} p \rightarrow \square_{R} \square_{R} p\right)$ and $\mathbf{5}\left(\diamond_{R} p \rightarrow \square_{R} \diamond_{R} p\right)$ axioms for all restricted modalities, resulting in the collapse of all iterated restricted modalities. Given S5 for $\square,\left(\square_{R-}\right.$ rig) also entails the metaphysical non-contingency of restricted modality $\left(\square \diamond_{R} p \vee \square \diamond_{R} p\right.$ and

\footnotetext{
12 This doesn't exactly get the logical form right, for several reasons, the least subtle one being that set theory isn't logic. If we want $\square \exists ! q R(q)$ to come out as a logical truth, we'll have to resort to higher-order modal logic. The full higher-order analysis is beyond the scope of this paper.
} 
$\square_{R} p \vee \square \square \square_{R} p$ ). Both consequences are unacceptable. The restricted modal claims we make outside of philosophical contexts are typically metaphysically contingent, and iterations of the restricted modal operators we typically use are not vacuous. $\left(\square_{R \text {-rig }}\right)$ is an acceptable analysis of actual restricted necessity, at least up to a standard of necessary equivalence: when we replace $\square_{R} p$ in ( $\square_{R \text {-rig }}$ ) with $@ \square_{R} p$ ('It is actually restrictedly necessary that $p$ '), the two sides of ( $\square_{R \text {-rig }}$ ) become necessarily equivalent, because it is a non-contingent matter which proposition is actually the restricting condition.

$\left(\square_{R}\right)$ gives restricted modalities a very weak logic: in particular it gives them the weakest normal modal logic, $\mathbf{K} .^{13}$ This is the result we want, since we assume (see note 4 ) that all objective modalities can be characterized by a relational semantics, and $\mathbf{K}$ is the strongest logic obeyed by all modalities that can be characterized by a relational semantics.

\section{Knowledge of restricted modality}

Let us now consider some epistemological consequences of the validity of each of $\left(\diamond_{R}\right)$ and $\left(\square_{R}\right)$. First, if one knows one side of the biconditional as well as the biconditional itself, one can come to know the other side by deducing it from these items of knowledge. Second, whether or not one knows the biconditional, one can come to know one side by deducing it from the other. Third, one can come to know one side without performing any deduction, simply by evaluating it by whatever method one could use to acquire knowledge of the other side.

The first generalization stands in little need of argument: knowledge can be extended by deduction. ${ }^{14}$ The second and third are no less plausible upon examination.

As regards the second generalization, coming to know $p$ by deducing it from $q$ does not require knowledge of any conditionals connecting $q$ and $p$.

As regards the third generalization, it is often the case that, when a deduction can extend knowledge of $p$ acquired by a certain method to knowledge of $q$, that same method can be employed to produce knowledge of $q$ directly. ${ }^{15}$ Recall the analogy with quantification. Even if one typically comes to know that there are (in an unrestricted sense) black squirrels in Canadian province $x$ by first coming to know that there are (in a sense restricted to $x$ ) black squirrels and then performing a deduction, there is no obstacle to one's skipping the provincially restricted knowledge and the deduction and coming to know that there are (unrestrictedly) black squirrels by whatever method one typically comes to have the provincially restricted knowledge. The converse is equally plausible. And the case of restricted modality is not relevantly different.

The deep structural analogy between restricted modality and restricted quantification bears emphasis here. Although the analogy is not controversial, it does not wear its epistemological significance on its sleeve.

Like modal operators, quantifiers are normally implicitly restricted by (non-trivial) conditions supplied by the context of speech. There is no beer-in a contextually restricted sense - not because there is no beer in the universe, but because there is no beer in your home (or whatever the relevant restricting property is). Restricted quantifiers are analyzable in terms

\footnotetext{
${ }^{13}$ With whatever restrictions to necessitation are mandated by the presence of the actuality operator @ and other indexicals in the language. By $\left(\square_{R}\right)$, necessitation for $\square_{R}$ inherits these restrictions from necessitation for $\square$.

${ }^{14} \mathrm{Of}$ course it does not follow that every possible deduction extends knowledge. Knowledge-extending deductions must be 'competent' (see Williamson 2000: 117, Hawthorne 2004: 34-35).

${ }^{15}$ There may be exceptions. Consider a case in which one comes to know a highly non-trivial mathematical fact $p$ by deducing it from some known axioms. We certainly don't want to claim that it is possible to come to know $p$ simply by doing whatever one actually did to come to know the axioms and then judging that $p$ on that basis. Thanks to Catharine Diehl for discussion here.
} 
of unrestricted quantifiers as follows, where $(\exists x: R(x))$ is a universal quantifier restricted by $R$.

$$
(\forall x: R(x)) F(x) \leftrightarrow \forall x(R(x) \rightarrow F(x))
$$

By the duality of the restricted quantifiers, we also have:

$$
(\exists x: R(x)) F(x) \leftrightarrow \exists x(R(x) \wedge F(x))
$$

Now suppose we are after a story about how we can know some restrictedly quantified claim to be true. Given the above equivalences, such a story will fall out of an account of how we can know the equivalent unrestrictedly quantified claim to be true, if we have such an account. One can come to know whether there is, in the 'in my home'-restricted sense, no beer by using any cognitive mechanisms by which one can come to know whether there is, in the unrestricted sense, something in one's home that is a beer. Of course, it is not inevitable that such a cognitive mechanism will always be (at least easily) available. Even though we have cognitive capacities that can deliver knowledge of some unrestrictedly quantified claims, they may in some cases be fairly useless for deciding restrictedly quantified claims, among other reasons because the restrictions in play are not transparent to us. For example, you may know that, in a certain restricted sense, there is no beer while having very little idea what property restricts your 'there is'. (The properties of being located in your refrigerator, of being located in your home, and of being a thing you own may all be equally plausible candidates.) In such a case you could not easily have come to know that there is, in just that restricted sense, no beer by whatever method you would evaluate the equivalent unrestricted 'there is' claim, because you are unable to make the restriction explicit.

Just as the standard restricted quantifiers can be analyzed in terms of unrestricted quantifiers, the restricting properties, and the truth-functional connectives, standard restricted modal operators can be analyzed in terms of metaphysical modality, restricting conditions (understood as having wide scope), and the truth-functional connectives. If we are after a story about how we can know some restricted modal claim to be true, then, given the equivalences provided by the analysis, such a story will fall out of an account of how we can know the equivalent unrestricted modal claim to be true, if we have such an account. Here, too, there is no guarantee that the cognitive mechanisms that deliver knowledge of unrestricted modality will always be (at least easily) available for deciding restricted modal claims. Here, too, the non-transparency of the restrictions may sometimes get in the way, as we will see.

\section{Extending knowledge of restricted modality by counterfactual reasoning}

In this section we will set aside the question 'How we can come to have any knowledge of restricted modality at all?' Instead we will ask: 'How, given that we do have some knowledge of restricted modality, can we extract further knowledge of restricted modality from it?'

There are, of course, many such ways, but here we will explore ones that exploit or are underwritten by logical relationships between restricted modal claims and counterfactuals. The following principle concerning counterfactuals and unrestricted modality is widely thought to be logically valid, ${ }^{16}$ and we will assume for now that it is (in $\S 5$ this assumption will face some complications).

$$
\text { PossibiLity } \quad(p \square \rightarrow q) \rightarrow(\diamond p \rightarrow \diamond q)
$$

\footnotetext{
${ }^{16}$ See Williamson (2007: 156), Lange (2009: 64), and Berto et al. (2017) for recent examples.
} 
PossibILITY is truth-functionally equivalent to this: if it is possible that $p$, and $p$ counterfactually implies $q$, then it is possible that $q$. In other words, PossiBILITY says that counterfactual modus ponens preserves possibility. Given that POSSIBILITY is valid, we can use our capacity for evaluating counterfactuals for extending our knowledge of both possibility and necessity. PossiBILITY underwrites a variety of ways to extend knowledge of $\diamond p$ and $p \square \rightarrow q$ to knowledge of $\diamond q$, as well as (by duality) to extend knowledge of $\square q$ and $\neg p \square \neg \neg q$ to knowledge of $\square p$. As in the case of the immediate epistemological applications of $\left(\square_{R}\right)$ and $\left(\diamond_{R}\right)$, for reasons that are by now familiar, these ways can but need not involve knowing PossibILITY, and they can but need not involve performing any deductions. (We will return to this last theme in $§ 5$.)

Similarly, we can use our capacity for evaluating counterfactuals for extending our knowledge of any restricted modality for which

\section{PossiBILITY* $^{*}(p \square q) \rightarrow\left(\diamond_{R} p \rightarrow \diamond_{R} q\right)$}

is valid. ${ }^{17}$ But here we face a problem: it is not entirely clear for which restricted modalities PosSIBILITY* is valid, and PosSIBILITY* is clearly not valid for some restricted modalities.

The validity of PossiBILITY* has been called into question even for one of our paradigmatic restricted modalities: nomic modality. On David Lewis's $(1973: 75,1979)$ view, which we think cannot be lightly dismissed, just about any departure from actuality would involve a violation of the laws of nature. The rough idea is that, for example, if you had had one more cup of coffee this morning than you actually did, then the history of the world up to your drinking that additional cup of coffee would have been as it actually is, whereafter it would have diverged - a 'local miracle' would have occurred. (This claim could be justified in terms of a Lewisian similarity-theoretic semantics for counterfactuals, but it need not be: entirely independently of any semantic theory, certain natural anti-'backtracking' judgments put a lot of pressure on one to draw Lewis's conclusion. ${ }^{18}$ ) But then, if the laws of nature are deterministic, something actually nomically impossible would have happened if you had had one more cup of coffee, etc. - a counterexample to PossIBILITY*, since it is nomically possible that you drink one more cup of coffee, etc. Even independently of determinism, it is plausible that the kinds of awkward transitions in world histories that the truth of various ordinary counterfactuals requires on Lewis's picture will sometimes be actually nomically impossible. And if there are such counterexamples to POSSIBILITY* for nomic modality, they will invalidate POSSIBILITY* for any more restricted modalities - so for pretty much any restricted modality we ordinarily express using the modal words. Consider the case of practical possibility. It is practically possible for Clinton to have won. But on the Lewisian picture, if Clinton had won, then she would have violated the actual laws of nature, and it is not practically possible for Clinton to violate the actual laws of nature.

Lewis's 'local miracle' view is controversial. But whether it is right or wrong, it should be uncontroversial that POSSIBILITY* is not valid for every restricted modality. For note that the condition that restricts a modal operator need not be true, in the sense that the proposition that satisfies $R$ in $\left(\square_{R}\right)$ may be false. So let $\diamond_{R}$ be restricted by a possibly true but actually false condition $r$, and let $p$ be any truth-functional tautology. Then $p \square \rightarrow \neg r$ and $\diamond_{R} p$ are both true, but $\diamond_{R} \neg r$ is false, so $(p \square \rightarrow r) \rightarrow\left(\diamond_{R} p \rightarrow \diamond_{R} \neg r\right)$ is a false instance of PossibILITY*.

Of course, this non-constructive argument for the existence of restricted modalities for

\footnotetext{
${ }^{17}$ Lange (2009: 64) endorses POSSIBILITY* for what he calls 'genuine' modalities. Genuine modalities are objective in the sense of this paper, but not vice versa (207-208, n. 5). According to Williamson (forthcoming), POSSIBILITY* is 'plausible for a wide range of restricted kinds of objective possibility'.

${ }^{18}$ See Dorr (2016b) for discussion.
} 
which PossibILITY* is not valid does not immediately undermine its epistemological applications (although it should make one worried). For all we have said so far, we never have occasion to think or talk about the restricted modalities for which POSSIBILITY* is not valid. But, in fact, we do think and talk about them often. Legal possibility, where the restricting condition is (roughly) that the relevant laws are obeyed, ${ }^{19}$ is a prominent example. ${ }^{20}$ Here is a direct counterexample to the validity of POSSIBILITY* for that restricted modality: It was legally possible for Nixon to win the 1968 Presidential election, and if Nixon had won the 1968 Presidential election, then Nixon would have ordered his subordinates to commit burglary (because he both won and ordered his subordinates to commit burglary), but it was not legally possible for Nixon to order his subordinates to commit burglary.

Where does this observation leave our hope to be able to use counterfactual thinking for extending our knowledge of restricted modalities? Happily, thanks to $\left(\square_{R}\right)$ and $\left(\diamond_{R}\right)$, POSSIBILITY makes available, at least in principle, ways of extending our knowledge of all restricted modalities by counterfactual reasoning. This is because PossiBILITY underwrites ways of projecting knowledge of what is actually restrictedly necessary (or possible) from knowledge of what is restrictedly necessary (or possible), and that something is actually restrictedly necessary (or possible) logically entails that it is restrictedly necessary (or possible).

Here is an example of one such way. Suppose that you know that $(i)$ it is nomically possible that you drop a certain piano, $P$, from a fifth-floor balcony. And suppose that you further know that, (ii) if you dropped $P$ from a fifth-floor balcony and the actual laws of nature obtained, then $P$ would shatter and the actual laws of nature would obtain. Then you can deduce from ( $i$ ) that it is (unrestrictedly) possible that the actual laws of nature obtain and you drop $P$ from a fifth-floor balcony. And you can further deduce from this and (ii), by PossiBILITY, that it is possible that $P$ shatters and the actual laws of nature obtain. Finally, you can deduce from this that the laws of nature are such that it is possible that they obtain and $P$ shatters - which is, by $\left(\diamond_{R}\right)$, equivalent to the claim that it is nomically possible that $P$ shatters. If you competently deduce this, you will know it, and so you will have used your capacity for evaluating counterfactuals for extending knowledge of nomic modality in a way underwriten by Possibility - and whether Possibility* is valid for nomic modality is neither here nor there.

When fully spelled out using our preferred formalization, the deduction just sketched has the following form.

$\begin{array}{ll}\text { 1. } & \diamond_{R} p \\ \text { 2. } & \exists r(@ R(r) \wedge r \wedge p) \square \exists r(@ R(r) \wedge r \wedge q) \\ \text { 3. } & \exists r(R(r) \wedge \diamond(r \wedge p)) \\ \text { 4. } & \exists r(@ R(r) \wedge \diamond(r \wedge p)) \\ \text { 5. } & \diamond \exists r(@ R(r) \wedge r \wedge p) \\ \text { 6. } & \diamond \exists r(@ R(r) \wedge r \wedge q) \\ \text { 7. } & \exists r(@ R(r) \wedge \diamond(r \wedge q)) \\ \text { 8. } & \exists r(R(r) \wedge \diamond(r \wedge q))\end{array}$

Assumption
Assumption
$1,\left(\diamond_{R}\right)$
3
4
2,5, POSSIBILITY
6
7

\footnotetext{
${ }^{19}$ Here we cannot, of course, simply think of the laws as what the law books explicitly dictate: what the law books explicitly dictate may be inconsistent, and therefore impossible to obey. The laws, rather, must be thought of as (in the typical case) a possible proposition determined by the explicit contents of legal texts and various features of the surrounding context, such as court decisions and perhaps the intentions of legislators.

${ }^{20}$ In common uses of 'legally possible' (one can find many examples by searching Google News for 'legally possible' together with 'Trump') the restricting condition is not that some relevant laws are obeyed. A typical restriction seems to concern particular people obeying laws with respect to particular actions and also to require that certain practical conditions obtain.
} 
The transitions from 3 to 4 and from 7 to 8 are justified by the logic of actuality ( $\varphi$ is equivalent to @ $\varphi$ ), and the transitions from 4 to 5 and from 5 to 6 are justified by the logic of necessity and actuality ( $\varphi$ is equivalent to $\square @ \varphi$ ). ${ }^{21}$ Similarly, one can use $\left(\square_{R}\right)$, the duality of $\diamond$ and $\square$, and the equivalence of $\exists q(R(q) \wedge \square(q \rightarrow p))$ with $\exists q(@ R(q) \wedge \square(q \rightarrow p))$ and of $\exists q(@ R(q) \wedge \square(q \rightarrow p))$ with $\square \exists q(@ R(q) \wedge(q \rightarrow p))$ to extend one's knowledge of restricted necessity by an argument underwritten by PossIBILITY.

By our observation (in $\S 2$ ) that $@ \diamond_{R} p$ is necessarily equivalent to $\diamond(r \wedge q)$, where $r$ is the condition that (actually) is $R$ (for example, $r$ is the conjunction of the actual laws of nature), one might hope to cut some corners by using the known counterfactual

$$
(r \wedge p) \square \rightarrow(r \wedge q)
$$

to extend one's knowledge of restricted possibility by PossiBILITY (and similarly, mutatis mutandis, for restricted necessity). In some cases this may be possible, but in general it seems to require some rather impressive cognitive achievements. For note first that, while $@ \diamond_{R} p$ and $\diamond(r \wedge p)$ are necessarily equivalent given that $r$ expresses the condition that satisfies $R$, they are not logically equivalent; their material equivalence follows by $\left(\diamond_{R}\right)$ from

$$
\exists ! q R(q)=r
$$

(' $r$ is the unique proposition with property $R$ '),

which in many cases will be quite difficult to know. In the case of nomic modality, knowing (!) requires knowing of a particular proposition, $r$, that it is the conjunction of the laws of nature, which would appear to be difficult. Second, suppose that, contrary to appearances, it is not difficult to know that $r$ is the conjunction of the laws of nature. (Perhaps there are easy, stipulative ways: 'Let ' $r$ ' express the conjunction of the laws of nature! Now I know that $r$ is the conjunction of the laws of nature.') Even if that is so, knowing something of the form ( $2 *)$ seems quite demanding. If one thinks $r$ under a fairly uninformative guise (e.g., 'Things are this way', where one somehow manages to refer to the conjunction of the laws of nature by 'this'), it is difficult to know what follows counterfactually from $r \wedge p$. If, on the other hand, one has a robust enough conception of the laws of nature to be able to know the relevant counterfactual, then that itself is a significant cognitive achievement. In contrast, it takes very little to know facts about how things would be if the actual laws of nature - whatever they may be- obtained and various other matters were otherwise. One has the latter kind of knowledge when one knows the premise

$$
\exists r(@ R(r) \wedge r \wedge p) \square \rightarrow \exists r(@ R(r) \wedge r \wedge q)
$$

Of course, knowing (2) is still in general a more impressive cognitive achievement than

\footnotetext{
${ }^{21}$ See the discussion of 'real-world validity' in Davies and Humberstone (1980), and Kaplan (1989: XVIII and 539: n. 65) on 'actually'. The 4-to-5 and 5-to-6 inferences also require the validity of the Barcan formulas for propositional quantifiers, which, in contrast with the first-order Barcan formulas, have tended not to be controversial. (In the recent debate on 'necessitism' sparked by Stalnaker (2012) and Williamson (2013), the validity of the propositionally quantified Barcan formulas has also been called into question (see Fritz 2016), but in the present dialectical context we take their validity to be sufficiently uncontroversial to assume without further commentary.)
} 
knowing $p \square \rightarrow q$. While it is fairly easy to know (2) when $R$ is the property of being the conjunction of the laws of nature, it may be much less easy in the case of various more ordinary restrictions - a theme to which we will return in the next section.

The key observation here is that, when it comes to using counterfactual reasoning for extending our knowledge of restricted possibility and necessity, the difference between restricted modality and actual restricted modality makes little difference. When we are after knowledge of what is restrictedly possible or necessary we can always use, mutatis mutandis, whatever means we have of coming to know that something is actually restrictedly possible or necessary to come to know that it is restrictedly possible or necessary, as long as no iterated modalities are involved. We only cannot in general use those means to come to know what would have been restrictedly possible or necessary had things been otherwise, or to come to know what is restrictedly necessarily necessary, or restrictedly necessarily possible, etc.

\section{Knowledge by imagination}

We often evaluate restricted modal claims by exercises of the imagination, and at least sometimes we acquire knowledge of restricted modality in this way:

Could we have hauled the piano upstairs, instead of taking it through the window? One might answer the question - indeed, one might come to know the answer-by imagining the piano being manipulated around the winding staircase. (2007: 136)

How might one come to know the answer in that way? Here, too, we can arrive at a plausible answer by reflecting on the epistemological significance of $\left(\square_{R}\right)$.

We assume, as is standard, that one of the cognitive mechanisms we use for obtaining knowledge of metaphysical modality is a certain type of imaginative exercise. ${ }^{22}$ To fix ideas, suppose that Williamson (2007: ch. 5) is right about the nature of these imaginative exercises. (A broadly similar story could be told using any of the competing accounts, but we use Williamson's as an illustration.) His account relies on the validity of $\left(\square_{\square}\right)$.

$(\square \rightarrow) \quad \square p \leftrightarrow(\neg p \square \mapsto \perp)$

As Williamson (2007: 155-158) observes, $(\square)$ is derivable from POSSIBILITY and the principle that strict implication is at least as strong as counterfactual implication $(\square(p \rightarrow q) \rightarrow(p \square \rightarrow$ $q))$ in $\mathbf{K} .\left(\diamond_{\square}\right)$ follows from $\left(\square_{\square}\right)$ by duality.

$\left(\diamond_{\square}\right) \quad \diamond p \leftrightarrow \neg(p \square \rightarrow \perp)$

On Williamson's view, the canonical way of evaluating a counterfactual is to suppose (counterfactually) that the antecedent holds, to develop that supposition using one's imagination - in effect, to imagine what else would be true if the antecedent were true - and to see whether such development 'robustly' yields the consequent or its negation. If it robustly yields the consequent, one accepts the counterfactual, and if it robustly fails to yield the consequent, one accepts its negation (2007: 152-155). If things go well, one thereby comes to know either the counterfactual or its negation. Thanks to the validity of ( $\left.\square_{\square}\right)$, we can come to know claims of metaphysical necessity and possibility by the same process of 'counterfactual development', either by evaluating the logically equivalent counterfactual or

${ }^{22}$ Contemporary defenses include Yablo (1993), Chalmers (2002), Gregory (2004), Williamson (2007: ch. 5) and Hill (2014). 
negated counterfactual and performing a deduction or by any of the other ways we have discussed of exploiting logical equivalences for acquiring knowledge.

By $\left(\square_{R}\right)$, this epistemological story is immediately applicable to restricted modalities. To evaluate $\square_{R} p$, one can evaluate the logically equivalent $\exists q(R(q) \wedge((q \wedge \neg p) \square \rightarrow \perp))$ by the canonical method for evaluating counterfactuals, and, if things go well, thereby arrive at knowledge of either $\square_{R} p$ or $\neg \square_{R} p$. (And similarly, mutatis mutandis, for $\diamond_{R} p$.) It is not easy, however, for things to go well in many typical cases. For familiar reasons, it is not straightforward, even in the case of nomic necessity, to know, concerning the conjunction $r$ of the laws of nature, what would be the case if $r$ and something else were the case. In that case, again, one can take a shortcut through the logic of actuality, asking instead what would be the case if the actual laws of nature, whatever they may be, obtained. But in the case of restrictions like those involved in various practical modal claims, this shortcut may not help. It does not seem easy to know what would be the case if the actual practical conditions, whatever they may be, obtained. How would one counterfactually suppose that the actual practical conditions, whatever they may be, obtain? The problem is that the second-order restricting condition $R$ is not, in many cases, any more transparent to thinkers of restricted modal contents than the condition $r$ that satisfies $R$.

Luckily, there is another way to use counterfactual suppositional reasoning to come to know restricted modal facts. It is even one that we commonly use for doing so: we often evaluate restricted modal claims by the canonical method for evaluating a counterfactual or a negated counterfactual that is restricted by the same condition. Like ordinary English modal operators, ordinary English counterfactuals are typically used, not for generalizing over absolutely all possibilities, but over the possibilities that satisfy a certain restriction. In Lewis's semantics, this restriction is represented, in effect, by an accessibility relation: an assignment 'to each world $i$ of $\ldots$ a set $S_{i}$ of worlds, regarded as the set of worlds accessible from $i$ ' (Lewis 1973: 48) — call this set the sphere of accessibility (around i) associated with $\square \rightarrow$ in the context. (Since counterfactuals embedded within other counterfactuals or within the scopes of modal operators are not at issue here, we will simply speak of 'the sphere of accessibility associated with $\square \rightarrow$ ' and ignore its world-relativity.) Roughly speaking, a counterfactual $p \square \rightarrow$ $q$ is true in a context just in case $q$ is true at all of the closest worlds within the sphere of accessibility supplied by the context at which $p$ is true. ${ }^{23}$ It follows that the equivalence

$\left(\square_{R_{\square}}\right) \quad \square_{R} p \leftrightarrow(\neg p \square \rightarrow \perp)$

and, by duality,

$\left(\diamond_{R_{\square}}\right) \quad \diamond_{R} p \leftrightarrow \neg(p \square \rightarrow \perp)$

will hold in any context in which the sphere of accessibility associated with $\square \rightarrow$ is the set of worlds in which the restriction associated with $\square_{R}$ is true. In such a context, to discover whether it is restrictedly necessary or possible that $p$, one can simply counterfactually suppose $p$ or its negation, and proceed to develop that supposition in imagination to see whether a contradiction follows. When one correctly evaluates a counterfactual by the canonical method, one's development in imagination of the supposition of the antecedent is constrained by the

\footnotetext{
${ }^{23}$ Less roughly, $p \square \rightarrow q$ is true at a world $w$ iff either (i) $p$ is not true at any world in $S_{w}$ or (ii) $p$ is true at some world $v$ in $S_{w}$ such that the material conditional $p \rightarrow q$ is true at every world that is at least as close to $w$ as $v$ (Lewis 1973: 49). Because Lewis is not concerned with indexicality, there is no explicit context parameter in his semantics, but the point of the assignment of spheres of similarity to worlds is to represent a restriction supplied by context.
} 
restriction associated with the counterfactual: one does not imagine possibilities that fall outside of the sphere of accessibility. This is how ordinary counterfactual reasoning proceeds when it proceeds correctly, and it requires no special cognitive achievements, such as supposing that a certain restriction holds, knowing what that restriction is, or even being able to describe it in any informative terms.

Let us now return to Byrne's piano example. Here, in more detail, is how one might come to know that the piano cannot be hauled upstairs through the stairway. One visually imagines the piano being moved through the stairway. In doing so one never visually imagines the piano beginning its journey with dimensions different from its actual dimensions, and one never visually imagines the piano changing its shape, or the stairway having dimensions different from its actual dimensions, and so on. Never visually imagining these things does not require one to know what the piano's or the staircase's dimensions are, or even to counterfactually suppose that the piano and the staircase have their actual dimensions, whatever they may be. One's attempts to develop the supposition by visually imagining it robustly fail-in effect, they lead to contradiction. (One need not explicitly derive a contradiction in order to detect a failure. It is often sufficient that one detects that the development is headed in an absurd direction: e.g., by being led to visualize parts of rigid bodies being superimposed.) On this basis one judges, and one comes to know, that the piano cannot be moved through the staircase.

In a case like the above, one typically does not come to know, because one does not come to believe, the restricted counterfactual $p \square \rightarrow \perp$. Rather, one simply forms the belief that $\neg \diamond_{R} p$ by the same method by which one would canonically evaluate $p \square \rightarrow \perp$, and that, together with the matching restriction being associated with $\square \rightarrow$ and $\diamond_{R}$, is sufficient for knowledge that $\neg \diamond_{R} p$. (In fact, there are good reasons not to explicitly consider $p \square \longmapsto \perp$, since this tends to shift the context: see below.)

Similarly, the kinds of extensions of restricted modal knowledge underwritten by PossibILITY and PossiBILITY* discussed in $\S 4$ need not involve ever coming to know, or even making a judgment on, a counterfactual. For example, a natural way to get to know that one can $A$ is to imagine oneself trying to $A$-with one's imagining restricted by the condition associated with 'can'-finding that one then imagines oneself succeeding, and judging on that basis that one can $A$ (cf. Williamson 2016a: 116). Here one extends one's knowledge that one can try to $A$ to knowledge that one can $A$ in a way underwritten by PossiBILITY*. But one does so without ever coming to know the relevant counterfactual. In a context in which PossiBILITY* holds, one can extend one's knowledge that $\diamond_{R} p$ to knowledge that $\diamond_{R} q$ directly by the canonical method for evaluating the counterfactual $p \square \rightarrow q$. And POSSIBILITY* does hold in any context in which the sphere of accessibility associated with $\square \rightarrow$ includes only possibilities in which the restricting condition associated with $\diamond_{R}$ is true. It is plausible that the contexts in which we find it natural to attempt to extend our knowledge of restricted modality in ways underwritten by POSSIBILITY* are also ones in which the restrictions of restricted modal operators and of counterfactuals are coordinated in this way.

We have just touched upon a feature of POSSIBILITY* that we ignored in $\S 4$ : the contextsensitivity of its antecedent. One should not simply ask whether POSSIBILITY* is valid for a particular restricted modality. Even for restricted modalities for which it is not valid, POSSIBILITY* may hold in some contexts. And it follows from what has been said that POSSIBILITY* does hold in every context, regardless of the restricted modality, in which the sphere of accessibility associated with $\square$ includes only possibilities in which the restricting condition associated with $\diamond_{R}$ is true. 
Does this mean that we can escape the strictures of $\S 4$ 's context-insensitive discussion and use POSSIBILITY* for extending knowledge of any restricted modality whatsoever simply by ensuring that we are in a context in which $\square \rightarrow$ is suitably restricted?

In fact, it does not. For a variety of restricted modalities, there are no suitable contexts. This is so for the simple reason that the sphere of accessibility associated with $\square \rightarrow$ must always include the world of the context, whereas the restricting conditions of many modal operators are not true in the world of the context. One cannot, for example, restrict $\square \rightarrow$ to worlds in which no violations of the penal code occur. If one could, one would thereby produce counterexamples to some of the most basic principles of counterfactual logic, including modus ponens. $^{24}$

The difference between restricted modalities that obey the $\mathbf{T}$ axiom $(\square p \rightarrow p$ ) and others seems to be significant here. While non-T restricted modalities cannot satisfy PossibILITY* no matter how we try to shift the context, there is no obvious reason why every T-obeying restricted modality could not satisfy POSSIBILITY* in some context. If so, PossibILITY* may have broader applications to the epistemology of modality than the discussion of $\S 4$ suggests.

Finally, it's worth noting that the context-sensitivity of the right side of Williamson's equivalence

$$
\left(\square_{\square}\right) \quad \square p \leftrightarrow(\neg p \square \rightarrow \perp)
$$

introduces a certain complication to his approach to the epistemology of metaphysical modality. It is this: ( $\square_{\square}$ ) holds only in contexts in which the sphere of accessibility associated with $\square \longrightarrow$ includes all possibilities (Strohminger and Yli-Vakkuri 2017: 833). Strictly speaking, then, we should not think of $\left(\square_{\square}\right)$ as logically valid. What is valid on Williamson's approach, rather, is

$\left(\square_{\square}{ }^{*}\right) \quad \square p \leftrightarrow\left(\neg p \square \rightarrow \lambda_{p \cdot p={ }_{\top}} \perp\right)$,

where $\square \rightarrow_{R}$ is a counterfactual conditional connective restricted to the set of possibilities in which the unique proposition $r$ such that $R(r)$ is true. ${ }^{25}$ Because the tautology $\mathrm{T}$ is true in all possibilities, the counterfactual in $\left(\square_{\square} *\right)$ generalizes over all possibilities. When we are not idealizing away the context-sensitivity of counterfactuals, the correct derivation of the Williamsonian equivalence $\left(\square_{\square}{ }^{*}\right)$ proceeds from

$\operatorname{NECESSITY}_{R} \quad \square(p \rightarrow q) \rightarrow\left(p \square \rightarrow \lambda_{p \cdot p={ }_{\top}} q\right)$

and

$\operatorname{PosSIBILITY}_{R}\left(p \square \lambda_{\lambda . p={ }_{\top}} q\right) \rightarrow(\diamond p \rightarrow \diamond q)$

in K. While Williamson's derivation of $\left(\square_{\square}\right)$ from NeCESSITY and PossiBILITY is valid, POSSIBILITY holds only in some contexts - namely, those in which the counterfactual conditional is unrestricted or, equivalently, restricted by a trivial condition as in $\operatorname{NECESSITY}_{R}$

\footnotetext{
${ }^{24}$ Let $r$ be the false but possible proposition that no violations of the (actual) penal code occur, and let $p$ be any truth-functional tautology. Suppose that $\square \rightarrow$ is restricted to worlds in which $r$ is true. Then $p$ and $p \square \rightarrow r$ are true but $r$ is false.

${ }^{25}$ If that proposition is true; otherwise it is restricted to the empty set.
} 
and $\operatorname{PossibILITY}_{R}$. For if any possibility $w$ falls outside its restriction, there will be at least one proposition $p$-namely $\{w\}$ - such that $(p \square \rightarrow \perp) \rightarrow(\diamond p \rightarrow \diamond \perp)$ is false. PossibILITY, and therefore $(\square \rightarrow)$, is only valid if we treat $\square \rightarrow$ as a logical constant that expresses what in ordinary English would be expressed by a counterfactual with a trivial restriction.

However, there is also another approach to the original Williamsonian analysis ( $\square_{\square}$ ), which is to treat the $\square \rightarrow$ in it as an ordinary context-sensitive counterfactual and to endorse it in all and only contexts in which its restriction excludes no possibilities. This is easier than it might at first appear. As we have previously argued (Strohminger and Yli-Vakkuri 2017: 833-34), having an explicit contradiction as the consequent of a counterfactual tends to force a trivially restricted reading of it. Again, the analogy with restricted quantifiers is illuminating. It is not easy to get into a context in which 'Everyone is prepared for the exam' has a trivially restricted reading - normally it expresses something like: Everyone enrolled in the class is prepared for the exam. But adding an explicit trivial restriction tends to get one into a context in which the resulting sentence does have a trivially restricted reading: try interpreting 'Everyone in the universe is prepared for the exam' in such a way that the 'everyone' is restricted to those enrolled in the class. It isn't easy, even though in principle it should be possible: after all, computed in the way semantics textbooks instruct us, the resulting restriction is to:

$\{x \mid x$ is in the universe $\} \cap\{x \mid x$ is in the class $\}=\{x \mid x$ is in the class $\}$.

The mechanism of semantic processing, however, does not deliver an intersective reading when the explicit restriction is trivial (or, in general, less restrictive than the attempted implicit restriction). A similar mechanism appears to be at work in the processing of counterfactuals, where an explicitly trivially false consequent tends to force the counterfactual to be evaluated with a trivial restriction. If so, getting into a suitable context will not require much more than considering Williamson's $\left(\square_{\square}\right)$.

The foregoing observation also introduces a complication to the epistemological applications of both POSSIBILITY and PosSIBILITY*: unless we treat $\square \rightarrow$ as a logical constant, as described above, PossiBILITY is not valid because there are contexts in which at least one world is excluded by the restriction of $\square \rightarrow$, and POSSIBILITY* is not valid for any restricted modality $\diamond_{R}$ whose restriction fails to exclude at least one world excluded by the restriction of $\square \rightarrow$. And even if we do treat $\square \rightarrow$ as a context-insensitive logical constant, neither PosSIBILITY nor PosSIBILITY* have any immediate epistemological significance, because the counterfactual reasoning we carry out in natural language and in thought is done using (possibly trivially) restricted counterfactuals and not the envisaged context-insensitive logical constant $\square \rightarrow$.

We suggest that this problem is not as serious as it may seem. We have already noted that it is plausible that the contexts in which we find it natural to attempt to extend our knowledge of restricted modality in ways underwritten by PosSIBILITY* are ones in which the restrictions of restricted modal operators and of counterfactuals are coordinated so that every possibility excluded by the restriction of the counterfactual conditional is also excluded by the restriction of the modal operators. It is also plausible that we find the same coordination in contexts on which we find it natural to attempt to extend our knowledge of metaphysical modality in ways underwritten by PossibILITY. Contexts of the latter kind are almost exclusively philosophical ones in which metaphysical modality is at issue, in which our counterfactuals are trivially restricted. They are contexts in which we naturally say things like 'That would lead to a contradiction', and so the mechanism of semantic processing described in the previous paragraph is plausibly at work and will deliver a trivial restriction for $\square \longrightarrow$. 


\section{Acknowledgments}

This paper started out as Margot Strohminger's project. Juhani Yli-Vakkuri was recruited as a coauthor in the final stages of preparation for publication. Credit (and blame!) should be assigned accordingly. We would like thank Timothy Williamson for detailed comments on early drafts of this paper, as well as Johannes Brandl, Jessica Brown, Catharine Diehl, Peter Fritz, Christopher Gauker, Sören Häggqvist, John Hawthorne, Hannes Leitgeb, Julien Murzi, Christian Nimtz, Barbara Vetter, and audiences at the Munich Center for Mathematical Philosophy (MCMP) at the Ludwig Maximilian University of Munich, the Free University of Berlin, the University of Antwerp, the University of Edinburgh, the University of Salzburg, and Bielefeld University for helpful comments and discussions. This research was supported by the Alexander von Humboldt Foundation.

\section{References}

Berto, F., French, R., Priest, G., \& Ripley, D. 2017. Williamson on counterpossibles. Journal of Philosophical Logic, https://doi.org/10.1007/s10992-017-9446-x.

Bull, R., \& Segerberg, K. (1984). Basic modal logic. In D. Gabbay and F. Guenthner (Eds.), Handbook of Philosophical Logic. Volume II: Extensions of Classical Logic (pp. 1-88). Dordrecht: D. Reidel.

Byrne, A. (2007). Possibility and imagination. Philosophical Perspectives, 21, 125-144.

Chalmers, D. J. (2002). Does conceivability entail possibility? In T. S. Gendler and J. Hawthorne (Eds.), Conceivability and Possibility (pp. 145-200). Oxford: Clarendon Press.

Davies, M., and Humberstone, L. (1980). Two notions of necessity. Philosophical Studies, 38, 1-30.

Dorr, C. (2016a). 'To be $F$ is to be $G$ ', Philosophical Perspectives, 30: 39-134.

Dorr, C. (2016b). Against counterfactual miracles. Philosophical Review, 125, 241-286.

Fine, K. (2014). Permission and possible worlds. Dialectica, 68, 317-336.

van Fraassen, B. (1977). The only necessity is verbal necessity. Journal of Philosophy, 74, 71-85.

Fritz, P. (2016). 'Propositional contingentism', The Review of Symbolic Logic, 9: 123-142.

Goodman, J. (2017). 'Reality is not structured', Analysis, 77: 43-53.

Gregory, D. (2004). Imagining possibilities. Philosophy and Phenomenological Research, 69, 327-348.

Hale, B. (2013). Necessary Beings: An Essay on Ontology, Modality, and the Relations Between Them. Oxford:

Oxford University Press.

Hale, B., \& Leech, J. (2017). Relative necessity formulated. Journal of Philosophical Logic, 46, 1-26.

Hawthorne, J. (2004). Knowledge and Lotteries. Oxford: Oxford University Press.

Hill, C. S. (2014). Conceivability and possibility. In his Meaning, Mind, and Knowledge (pp. 273-296). Oxford: Oxford University Press.

Humberstone, I. L. (1981). Relative necessity revisited. Reports on Mathematical Logic, 13, 33-42.

Kaplan, D. (1989). Demonstratives. In J. Almog et al. (Eds.), Themes from Kaplan (pp. 481-563). Oxford:

Oxford University Press. Completed and circulated in mimeograph in the published form in 1977.

Kment, B. (2014). Modality and Explanatory Reasoning. Oxford: Oxford University Press.

Kratzer, A. (1981). The notional category of modality. In H.-J. Eikmeyer and H. Rieser (Eds.), Words, Worlds, and Contexts (pp. 38-74). Berlin: de Gruyter.

Kratzer, A. (2012). Modals and Conditionals. Oxford: Oxford University Press.

Lange, M. (2009). Laws and Lawmakers: Science, Metaphysics, and the Laws of Nature. New York: Oxford University Press.

Lewis, D. (1973). Counterfactuals. Oxford: Blackwell.

Lewis, D. (1979). Counterfactual dependence and time's arrow. Noûs, 13, 455-476.

Peacocke, C. (1999). Being Known. Oxford: Clarendon Press.

Portner, P. (2009). Modality. Oxford: Oxford University Press.

Stalnaker, R. (2012). Mere Possibilities. Oxford University Press.

Sainsbury, M. (1997). Easy possibilities. Philosophy and Phenomenological Research, 57, 907-919.

Strohminger, M. (2015). Perceptual knowledge of nonactual possibilities. Philosophical Perspectives, 29, 363375.

Strohminger, M., \& Yli-Vakkuri, J. (2017). The epistemology of modality. Analysis 77: 825-838.

Vetter, B. (2015). Potentiality: From Dispositions to Modality. Oxford: Oxford University Press.

Williamson, T. (2000). Knowledge and Its Limits. Oxford: Oxford University Press.

Williamson, T. (2007). The Philosophy of Philosophy. Oxford: Blackwell.

Williamson, T. (2016a). Knowing by imagining. In A. Kind \& P. Kung (Eds.), Knowledge Through Imagination (pp. 113-123). Oxford: Oxford University Press.

Williamson, T. (2016b). Modal science. Canadian Journal of Philosophy 46: 453-92. Reprinted in Yli-Vakkuri and McCullagh 2017. 
Williamson, T. (2016c). Reply to Sider. Canadian Journal of Philosophy 46: 796-802. Reprinted in Yli-

Vakkuri and McCullagh 2017.

Williamson, T. (forthcoming). The counterfactual-based approach to modal epistemology. In O. Bueno and S. Shalkowski (Eds.), The Routledge Handbook of Modality. London: Routledge.

Yablo, S. (1993). Is conceivability a guide to possibility? Philosophy and Phenomenological Research, 53, 142.

Yli-Vakkuri, J., \& Hawthorne, J. (MSa). Modal epistemology. Unpublished manuscript.

Yli-Vakkuri, J., \& Hawthorne, J. (MS $b$ ). The necessity of mathematics. Unpublished manuscript.

Yli-Vakkuri, J., \& McCullagh, M., eds. (2017). Williamson on Modality. London: Routledge. 